\title{
Una aproximación cartográfica al análisis de los vertidos de metales pesados en España
}

\author{
A cartographic approach to the analysis of \\ heavy metal spills in Spain \\ Fabián García Hernández ${ }^{1}$ \\ Francisco Escobar Martínez²
}

Recibido 9 de abril de 2020; aceptado 14 de junio de 2020

\section{RESUMEN}

A pesar de los demostrados efectos adversos que los metales pesados (MP) provocan en la salud humana, su distribución espacial en España es un fenómeno poco estudiado. Con la finalidad de paliar esta deficiencia, se plantearon en esta investigación los siguientes objetivos: 1) conocer la distribución espacial, a escala municipal, de los vertidos de MP emitidos a las aguas, 2) estimar y cartografiar la peligrosidad de los vertidos según su toxicidad, 3) comparar las emisiones de MP en España con su entorno europeo y 4) estimar la evolución futura de los vertidos de MP. Los datos se han recopilado desde el registro E-PRTR (European Pollutant Release and Transfer Register 2007-2016) y la localización de los puntos de emisión se ha obtenido mediante geocodificación. Tras un análisis exploratorio, los datos fueron normalizados y ponderados, facilitando la interpretación de gráficas, tablas y cartografía. Los resultados muestran una mayor concentración de puntos de emisión en los sectores norte (Asturias, Cantabria y País Vasco), nordeste (Cataluña), este-sureste (Valencia) y sur (Andalucía). Tras revisar los efectos de los MP sobre la salud humana, estos han sido ponderados por este orden; mercurio $(\mathrm{Hg})$, plomo $(\mathrm{Pb})$, cadmio $(\mathrm{Cd})$, cromo ( $\mathrm{Cr}$ ), arsénico (As) y zinc ( $\mathrm{Zn}$ ). Se ha constatado que durante el periodo 2007-2016, las cantidades de MP vertidas a las aguas lejos de disminuir, han oscilado adaptándose a las transiciones económicas y persistiendo una mayor 
concentración de vertidos en los municipios localizados en las provincias del norte, nordeste, este-sureste y sur del país.

Palabras clave: metales pesados, vertidos, emisión, distribución espacial, cartografía aplicada.

\section{ABSTRACT}

Despite the proven adverse effects that heavy metals (HM) cause on human health, their spatial distribution in Spain remains under studied. In order to alleviate this deficiency, the following objectives were set: 1) to know the spatial distribution, at local scale, of HM released into stream waters, 2) to estimate and map the danger of the spills according to their toxicity, 3) to compare $\mathrm{HM}$ emissions in Spain within the European context and 4) to estimate future evolution of HM spills. Data has been collected from the E-PRTR (European Pollutant and Transfer Register, 2007-2016) and the location of the emission points has been obtained by geocoding. After exploratory analysis, data were normalized and weighted, facilitating the interpretation of graphs, tables and cartography. Results show a higher concentration of emission points in the North (Asturias, Cantabria and Basque Country), Northeast (Catalonia), East-Southeast (Valencia) and South (Andalusia) sectors. After reviewing the effects of HMs on human health, they have been weighted in this order; mercury $(\mathrm{Hg})$, lead $(\mathrm{Pb})$, cadmium (Cd), chromium (Cr), arsenic (As) and zinc (Zn). It has been verified that during the period 2007-2016, the quantities of PM discharged into the waters, far from decreasing, have fluctuated, adapting to economic transitions and persisting a greater concentration of discharges in the municipalities located in the northern, northeastern, southeastern and southern provinces.

Key words: heavy metals, spills, emissions, spatial distribution, cartographic analysis.

\section{Introducción}

En la literatura científica se aprecia un interés creciente por el análisis de los efectos que la presencia de metales pesados en el medio ambiente ejercen sobre la salud humana (Morais et al., 2012; Atta et al., 2016; Sánchez-Díaz et al., 2018), sobre el medio ambiente y sobre la alimentación (Cuihua Chen et al., 2007). La toxicidad (Achparaki et al., 2012; Moslen y Miebaka, 2018) de estos elementos y sus repercusiones en la salud (Peng et al., 2011; Jaishankar et al., 2014) se han estudiado partiendo de diferentes perspectivas teniendo en cuenta no solo cómo afectan a la población sino también cómo es absorbida por esta y cuáles son los puntos de origen (Londoño-Franco et al., 2016; Moslen y Miebaka, 2018). La Unión Europea ha aprobado la directiva 2010/75/EU encaminada a establecer umbrales límites de vertidos y a llevar un registro de las emisiones tanto a las aguas como al aire (EU, 2010). En lo que se refiere al ámbito español, son escasos los estudios enfocados exclusivamente al análisis de la distribución espacial de los MP aunque sí hay estudios que analizan la relación entre los 
vertidos de MP a los ríos y determinadas enfermedades (Sánchez-Díaz et al., 2018).

La normativa europea (European Commission, 2006; EU, 2010) establece una serie de medidas destinadas a limitar los vertidos industriales de MP. Entre ellas, se han fijado los máximos autorizados a cada empresa. La unidad registrada son kilogramos (kg) por año. Las empresas deben comunicar sus emisiones de forma obligatoria únicamente cuando el umbral máximo establecido ha sido superado (Comisión Europea, 2006). La Tabla 1 recoge los umbrales de emisión para los 6 MP más comunes.

Tabla 1. Umbrales límite en las emisiones de MP

\begin{tabular}{cc}
\hline Metal pesado & Umbral \\
\hline Mercurio $(H g)$ & 1 kg/año \\
\hline Plomo $(\mathrm{Pd})$ & $20 \mathrm{~kg} / \mathrm{año}$ \\
\hline Cadmio $(\mathrm{Cd})$ & $5 \mathrm{~kg} / \mathrm{año}$ \\
\hline Cromo $(\mathrm{Cr})$ & $50 \mathrm{~kg} / \mathrm{año}$ \\
\hline Arsénico $(\mathrm{As})$ & $5 \mathrm{~kg} / \mathrm{año}$ \\
\hline Zinc $(\mathrm{Zn})$ & $100 \mathrm{~kg} /$ año
\end{tabular}

Fuente: E-PRTE.

Debido a la multiplicidad de efectos y factores involucrados, no existe un consenso en la determinación cuantitativa del grado de peligrosidad de los MP, aunque sí se han publicado aproximaciones basadas en diferentes variables. Son notables los estudios que analizan los efectos que los MP ejercen sobre la salud humana (Yu, 2005; Morais et al., 2012; Jaishankar et al., 2014), sobre el medio ambiente (Cuihua Chen et al. 2007, Tchounwou et al., 2012; Ismail et al., 2013; Sánchez-Díaz et al., 2018), sobre cómo se distribuyen en el medio natural (Sánchez-Díaz et al., 2018), o su transferencia a la flora y la fauna (LondoñoFranco et al., 2016). No obstante, sí existe un consenso acerca de qué variables son las que determinan la peligrosidad de los MP y se relacionan con el tiempo de exposición, el grado de concentración, la cercanía a los mismos y al tipo de químico principalmente.

Desde un enfoque marcadamente cartográfico, los objetivos que guiaron el trabajo fueron:

- Cuantificar la peligrosidad de los vertidos según su toxicidad y producir una cartografía de áreas con mayor concentración de MP.

- Comparar las emisiones de MP de España con las de los países de su entorno europeo.

- Conocer la distribución espacial de los puntos de emisión y determinar la cantidad de MP que se vierten por año.

- Estudiar la evolución de los vertidos de MP. 


\section{Métodos}

\subsection{Zona de estudio}

El ámbito de estudio es el territorio nacional español. España cuenta con una industria diversa que combina industria tradicional y moderna distribuida de forma irregular por el territorio, concentrando los principales núcleos industriales en Cataluña, Comunidad Valenciana, País Vasco y Madrid. Esto hace que las emisiones de MP se distribuyan por casi toda la geografía española, aunque su intensidad sea más potente en áreas localizadas en el norte y en el arco mediterráneo, coincidiendo con áreas densamente pobladas. De cara a contextualizar la situación de España a nivel europeo se ha recogido información de cada uno de los países que forman parte de la E-PRTR para producir una cartografía comparativa.

\subsection{Unidades de observación}

Partiendo de los puntos emisores como unidad de observación primaria, se han agregado los datos a nivel municipal y provincial y, además, se han descargado y cartografiado datos a nivel de país por lo que la unidad de observación es múltiple: punto de emisión, local, provincial y nacional. El análisis a diferentes escalas nos ha permitido observar la distribución general de los puntos de emisión, localizar las áreas con mayor concentración de MP y realizar una comparación de España a nivel europeo.

\subsection{Datos}

Los datos recopilados provienen de tres fuentes principales:

1. European Pollutant Release and Transfer Register (E-PRTR). A través de la web oficial de la E-PRTR, se ha accedido a la base de datos donde se recogen los registros de vertidos de MP a las aguas (E-PRTR Datasets, diffuse emissions to water) y a la legislación que regula la emisión de vertidos a nivel europeo (Comisión Europea, 2006). Los datos de vertidos de MP son compilados por las autoridades competentes de cada país y facilitadas a la Comisión Europea y a la Agencia Europea del Medio Ambienta (EEA en sus siglas en inglés) quienes copilan, revisan y difunden los datos a través de la web E-PRTR para todo el mundo.

2. Instituto Geográfico Nacional (IGN) y Natural Earth. A través del Centro de Descargas del Centro Nacional de Información Geográfica (CNIG) del Instituto Geográfico Nacional (IGN), se ha obtenido la cartografía base a escala 1:3000 000 en formato shapefile para representar las diferentes unidades administrativas de España. En el caso de Europa, se ha descargado la cartografía base a escala 1:50 000000 en formato shapefile que se encuentra disponible en el portal de descargas de la web de Natural Earth.

3. World Bank Open Data / Banco Mundial de Datos (BMD). Desde la web del Banco Mundial de Datos se han descargado los datos relativos al Índice de 
Producción Industrial de España basado en la tasa de crecimiento anual (valor agregado industrial) para el periodo de 2007 a 2016.

\subsection{Técnicas aplicadas}

\subsubsection{Filtrado de datos}

Para analizar los datos correctamente ha sido necesario filtrar la base de datos. En un primer filtrado se han seleccionado únicamente los datos relativos a las emisiones de España, excluyéndose el resto de países europeos. En un segundo filtro se han seleccionado las emisiones a las aguas y se han excluido las emisiones al aire. Finalmente, con un tercer filtro se han seleccionado los datos del año 2016, la fecha disponible más actual. La serie de años analizada coincide con el periodo en el que hay datos disponibles, desde 2007 hasta 2016.

\subsubsection{Depuración de datos}

Tras el filtrado, se observaron repeticiones en los datos por lo que fue necesario ordenarlos por empresa y tipo de metal para detectarlos y posteriormente eliminarlos. A su vez, se ha intentado seleccionar una empresa por cada tipo de metal que haya realizado el registro de MP vertidos a las aguas todos los años, entre 2007 y 2016, para evaluar su tendencia evolutiva. De los MP analizados, se logró para el cromo, cadmio, mercurio y zinc al menos una empresa con registros para los 10 años de la serie. En el caso del arsénico y el plomo no hay ninguna empresa con datos para toda la serie analizada. Aunque lo ideal hubiera sido contar con el conjunto de todas las empresas declarantes, se ha seleccionado únicamente una empresa por MP debido a la frecuente ausencia de datos en muchos de los años de la serie. Apenas un total de entre tres y cinco empresas han declarado en todos y cada uno de los años en los que se ha desarrollado el registro de emisiones de MP, lo cual dificulta realizar un seguimiento individualizado por empresa. Teniendo esto en cuenta, se han escogido dos empresas que tienen registros para la totalidad de los años analizados exceptuando los registros de 2007 y 2008 para el arsénico y 2007, 2013 y 2014 para el plomo. Para paliar estos huecos en la serie, se ha realizado un promedio con los años en los cuales contamos con datos para obtener un valor aproximado.

\subsubsection{Ceocodificación, espacialización y agregación}

Una vez filtrados y depurados los datos se ha procedido a espacializar aquellos correspondientes al año 2016 mediante geocodificación. Los datos obtenidos de la E-PRTR albergan la latitud y longitud de los puntos en donde las empresas han realizado los vertidos. El proceso se realiza mediante el software ArcGIS, donde en primer lugar, se importan los datos desde el archivo Excel de vertidos, $y$, en segundo lugar, se proyectan en el sistema de referencia ETRS_1989_UTM_ Zona_30N. Se ha aplicado este sistema de referencia en vez del sistema de referencia ETRS-Cónica Conforme de Lambert debido a que la investigación 
se enfoca exclusivamente en la península ibérica y no se incluye a Canarias ya que no hay datos para esta región. Los datos espacializados se representan mediante un punto caracterizado por los atributos de emisiones relativos a una empresa en una localización exacta por lo que, de cara a su posterior representación y análisis a nivel municipal, es necesario realizar una agregación de estos a las unidades municipales. A modo de aclaración se debe mencionar que tras contactar con la fuente de los datos (Registro Estatal de Emisiones y Fuentes Contaminantes, Ministerio para la Transición Ecológica) se confirma que las coordenadas utilizadas para referenciar los vertidos de MP establecidas en la base de datos descargada inicialmente hacen referencia al punto de emisión de vertidos y no la razón social como se creía en un principio lo cual ofrece mayor precisión y rigor a los análisis.

\subsubsection{Normalización y ponderación}

Para facilitar la comparación y la correcta representación de los datos, se han aplicado dos procesos previos. Por un lado, debido a que los valores registrados de los MP son muy dispares, se han normalizado aplicando primero la fórmula 1;

$$
z=\frac{x-\min (x)}{\max (x)-\min (x)} * 100
$$

donde $x$ es el rango de total de valores, $\min (x)$ es el valor mínimo de $x, \max (x)$ es el valor máximo de $x$,

y, posteriormente, se aplicó la fórmula 2;

$$
z=\frac{x i-\mu}{\sigma}
$$

Por otro lado, ha sido necesario ponderar los valores relativos a los vertidos de MP a las aguas a la hora de representarlos en un mapa. Esto se debe a que cada municipio puede albergar una o varias empresas o puntos de emisión, y a su vez, a que cada empresa puede emitir uno o más tipos de metal cuya consideración de peligrosidad también varía según el tipo metal. Para solventar este inconveniente y obtener un único valor para cada municipio se ponderaron los valores de emisión aplicando la fórmula 3;

$$
P O N=\left(m p 1 * p 1+m p 2 * p 2+m p 3^{*} p 3+m p 4 * p 4+m p 5^{*} p 5+m p 6^{*} p 6\right)
$$

donde se realiza el sumatorio obtenido de multiplicar el valor de cada uno de los metales pesados $m p$ por su peligrosidad $p$ dividido entre la cantidad total de metales.

Al igual que en el caso de los municipios, para poder comparar los datos de España con los de los países europeos en el año 2016, se ha aplicado la misma ponderación a nivel nacional. De este modo, se adopta el porcentaje 
de emisiones que realiza cada país con respecto al total por tipo de metal y se aplica la fórmula de ponderación (3) para determinar qué países presentan un grado de peligrosidad bajo o alto según la cantidad de metales que concentran. Un ejemplo sería el siguiente:

\begin{tabular}{lccccccc}
\hline $\begin{array}{c}\text { País } / \\
\text { metal } \\
\text { (peso) }\end{array}$ & $\begin{array}{c}\text { Arsénico } \\
(20)\end{array}$ & $\begin{array}{c}\text { Cadmio } \\
(40)\end{array}$ & $\begin{array}{c}\text { Cromo } \\
(30)\end{array}$ & $\begin{array}{c}\text { Mercurio } \\
(60)\end{array}$ & $\begin{array}{c}\text { Plomo } \\
(50)\end{array}$ & $\begin{array}{c}\text { Zinc } \\
(10)\end{array}$ & Ponderación \\
\hline Serbia & 39,81 & 20,47 & 8,1 & 31,86 & 13,99 & 2,35 & 748,8 \\
\hline
\end{tabular}

$$
P O N=\left(39,81^{*} 20+20,47 * 40+8,1 * 30+31,86 * 60+13,99 * 50+2,35 * 10\right) / 6=748,8
$$

\subsubsection{Diseño de gráficas}

La representación visual de la evolución en la emisión de MP mediante gráficas permite determinar si ha habido una mejora o empeoramiento del fenómeno a lo largo del periodo estudiado. Dados los valores tan extremos que presentan los registros dependiendo del tipo de metal, se ha optado por un análisis individual. Para el análisis de tendencia se han agrupado los MP según su tipología y año de registro. Tras esto, se ha realizado un sumatorio de todas las cantidades por metal y año con la serie completa. En lo que se refiere a las gráficas de comparación de España con el resto de los países europeos, se han seleccionado los países que presentan un valor superior a 1\% evitando así la saturación de datos que dificulten la visualización.

\subsubsection{Representación cartográfica}

Los resultados se presentan en forma de mapa. Para representar la cantidad de MP por provincias y municipios se ha aplicado una clasificación en intervalos de progresión geométrica donde mediante las variables color, tamaño y forma se representa el tipo y la cantidad de MP por unidad espacial. Se ha aplicado el mismo método de clasificación de la variable temática para representar la cantidad total de vertidos por provincia. En este caso, la variable visual tamaño, es la adoptada para representar los valores absolutos agregados a nivel provincial. Por último, para representar el grado de peligrosidad, se ha aplicado una clasificación ordinal donde se representa mediante la variable visual valor el grado de peligrosidad que tiene cada unidad espacial, tanto a nivel local como europeo. Se han incluido gráficos de tartas que representan la cantidad de MP por tipo de metal que se concentra en una determinada área.

\section{Resultados}

\subsection{Efectos de los MP sobre la salud humana}

Teniendo en cuenta la información extraída de estudios previos sobre los efectos que generan los MP en la salud humana (Yu, 2005; Morais et al., 2012, Jaishankar 
et al., 2014), se propone un ranking de MP, construido a partir de la asignación de diferentes pesos, de peligrosidad de MP según su toxicidad (Tabla 2).

\subsection{Vertidos en Europa}

El grado de peligrosidad que se asocia a España según los valores de emisiones analizados para el 2016 es de medio-alto ocupando la octava posición de un total de 31 países. Los 10 países con mayor grado de peligrosidad, en orden descendente, son Serbia, Italia, Reino Unido, Polonia, Alemania, España, Francia, Bulgaria, Portugal y Finlandia (Tabla 3).

Tabla 2. Peligrosidad de los metales pesados según su toxicidad y efectos en la salud

\begin{tabular}{|c|c|c|c|c|}
\hline Ranking & $M P$ & Umbral & Peso & Efectos en la población \\
\hline $1^{\circ}$ & $\begin{array}{l}\text { Mercurio } \\
\quad(\mathrm{Hg})\end{array}$ & 1 kg/año & 60 & $\begin{array}{l}\text { Daños permanentes en el cerebro y } \\
\text { riñones, daños al feto en desarrollo, } \\
\text { irritabilidad, temblores, daños en la } \\
\text { visión y audición, problemas de memoria, } \\
\text { náuseas, vómitos, gastroenteritis, daño } \\
\text { en los pulmones, aumento de la presión } \\
\text { arterial, erupciones en la piel, irritación } \\
\text { ocular, muerte }\end{array}$ \\
\hline $2^{\circ}$ & $\begin{array}{l}\text { Plomo } \\
\text { (Pd) }\end{array}$ & 20 kg/año & 50 & $\begin{array}{l}\text { Alteraciones de la biosíntesis de } \\
\text { hemoglobina y anemia, aumento presión } \\
\text { sanguínea, daños en los riñones, aborto } \\
\text { espontáneo, alteraciones del sistema } \\
\text { nervioso, esterilidad, debilitación de } \\
\text { capacidad de aprendizaje en niños, } \\
\text { hiperactividad, deterioro de la dentadura, } \\
\text { alteraciones graves del equilibrio, muerte }\end{array}$ \\
\hline $3^{\circ}$ & $\begin{array}{l}\text { Cadmio } \\
\text { (Cd) }\end{array}$ & 5 kg/año & 40 & $\begin{array}{l}\text { Daños renales, alteración del sistema } \\
\text { nervioso e inmunitario, alteración de las } \\
\text { células de AND, vómitos, daños óseos }\end{array}$ \\
\hline $4^{\circ}$ & $\begin{array}{l}\text { Cromo } \\
(\mathrm{Cr})\end{array}$ & 50 kg/año & 30 & $\begin{array}{l}\text { Alteración de células, aparición de } \\
\text { cáncer, trastornos metabólicos, daños } \\
\text { en el corazón, diabetes, fallos hepáticos, } \\
\text { alteración en los tejidos nerviosos, fallas } \\
\text { en el sistema circulatorio, úlceras de piel, } \\
\text { problemas renales }\end{array}$ \\
\hline $5^{\circ}$ & $\begin{array}{l}\text { Arsénico } \\
\text { (As) }\end{array}$ & 5 kg/año & 20 & $\begin{array}{l}\text { Alteraciones en el sistema nervioso, } \\
\text { riñones, aparato digestivo, epidermis, } \\
\text { riego sanguíneo, ceguera, anemia, } \\
\text { gastroenteritis, fiebres }\end{array}$ \\
\hline $6^{\circ}$ & $\begin{array}{l}\text { Zinc } \\
\text { (Zn) }\end{array}$ & 100 kg/año & 10 & $\begin{array}{l}\text { Daños en el estómago e infecciones de la } \\
\text { mucosa }\end{array}$ \\
\hline
\end{tabular}


Tabla 3. Ranking de países según cantidad de MP emitidos ordenados por el grado de peligrosidad (2016)

\begin{tabular}{|c|c|c|c|c|c|c|c|}
\hline \multirow{2}{*}{ País } & \multicolumn{6}{|c|}{$M P(\%)$} & \multirow{2}{*}{ Ponderación } \\
\hline & Arsénico & Cadmio & Cromo & Mercurio & Plomo & Zinc & \\
\hline Serbia & 39,81 & 20,47 & 8,1 & 31,86 & 13,99 & 2,35 & 748,8 \\
\hline Italia & 15,04 & 22,42 & 30,28 & 12,01 & 24,63 & 8,97 & 691,3 \\
\hline R. Unido & 11,73 & 5,94 & 7,52 & 15,88 & 12,31 & 16,32 & 404,9 \\
\hline Polonia & 4,9 & 12,73 & 4,58 & 6,09 & 10,48 & 9,19 & 287,7 \\
\hline Alemania & 3,64 & 2,85 & 12,43 & 2,96 & 5,21 & 13,41 & 188,7 \\
\hline España & 2,5 & 3,79 & 3,91 & 8,39 & 3,41 & 4,33 & 172,7 \\
\hline Francia & 3,46 & 4,23 & 3,7 & 3,85 & 5,69 & 4,85 & 152,2 \\
\hline Bulgaria & 1,94 & 8,33 & 2,61 & 0,87 & 7,79 & 1,48 & 151,1 \\
\hline Portugal & 2,13 & 7,00 & 5,35 & 1,52 & 0,83 & 1,52 & 105,2 \\
\hline Finlandia & 1,51 & 2,03 & 2,73 & 1,52 & 1,83 & 3,36 & 68,3 \\
\hline
\end{tabular}

Los datos presentados en la Tabla 3 han sido cartografiados (Figura 1). Como era esperable, se aprecia cómo los países clasificados como de mayor grado de peligrosidad son los países industrialmente más desarrollados.

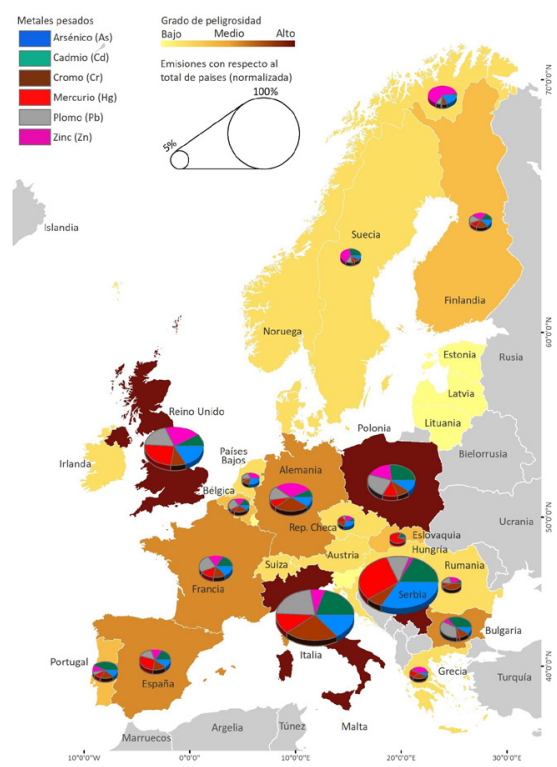

Figura 1. Grado de peligrosidad y cantidad de MP emitidos por los países miembros del E-PRTR. 
En cuanto al análisis individualizado por MP, España ocupa la octava posición en emisiones de cadmio (Cd) (Figura 2), arsénico (As) (Figura 3), y plomo $(\mathrm{Pb})$ (Figura 4), la décima en zinc (Zn) (Figura 5), la novena posición en emisiones de cromo ( $\mathrm{Cr}$ ) (Figura 6) y la cuarta posición en emisiones de mercurio (Hg) (Figura 7) presentando valores que oscilan entre el 2\% y $8 \%$ del total de emisiones que vierten el conjunto total de países.

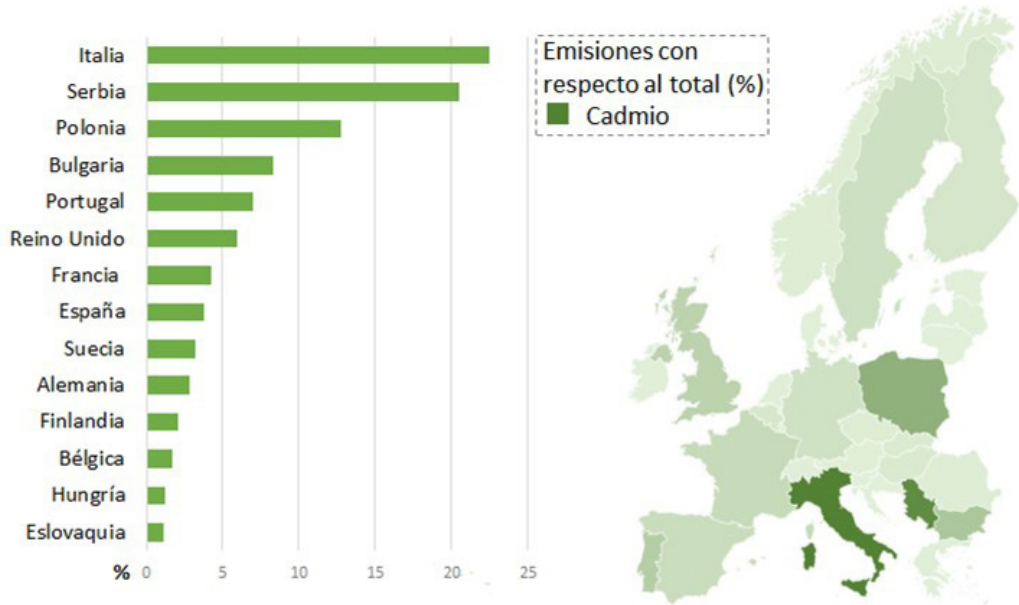

Figura 2. Emisiones de cadmio. Contenido obtenido de E-PRTR.

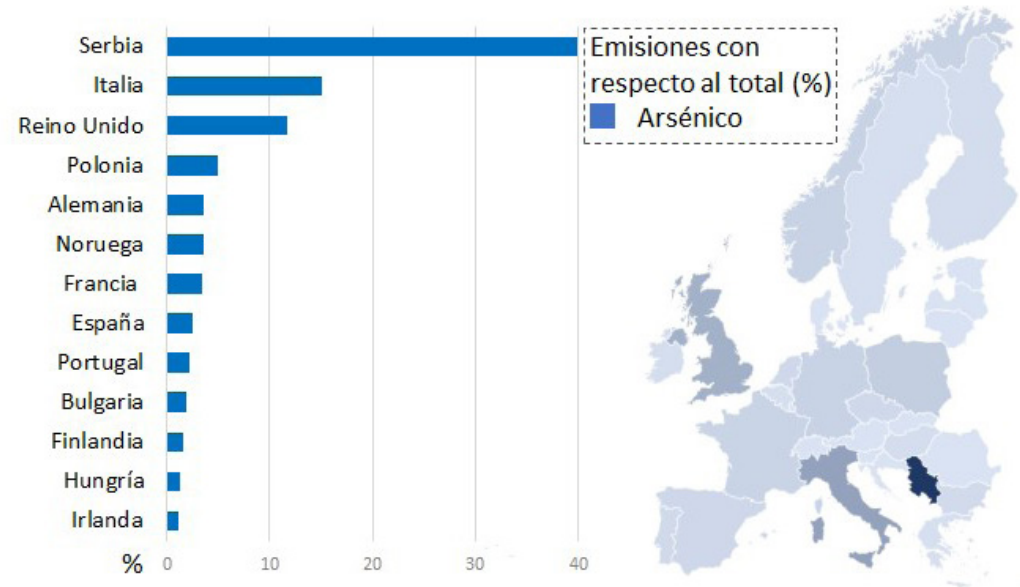

Figura 3. Emisiones de arsénico. Contenido obtenido de E-PRTR. 

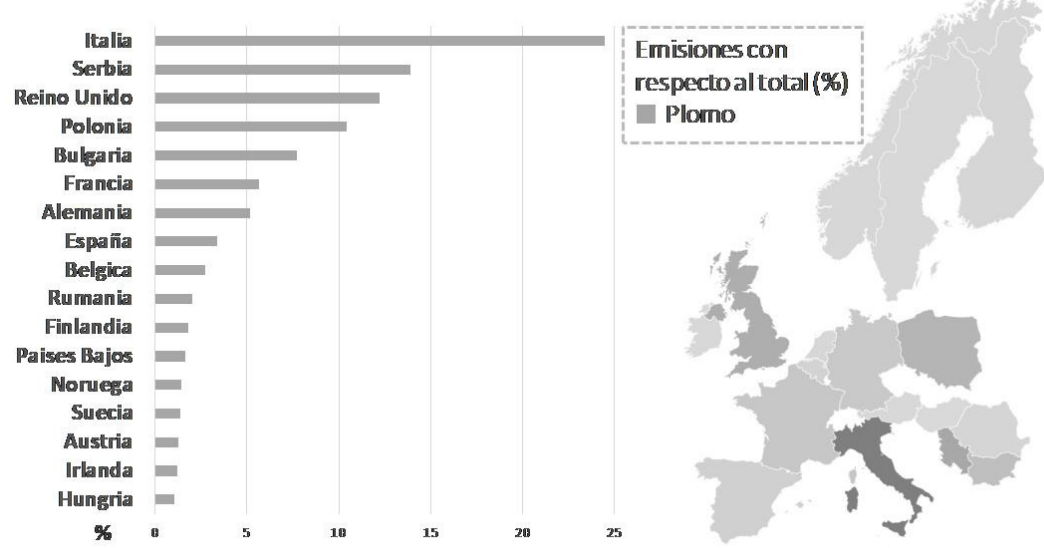

Figura 4. Emisiones de plomo. Contenido obtenido de E-PRTR.
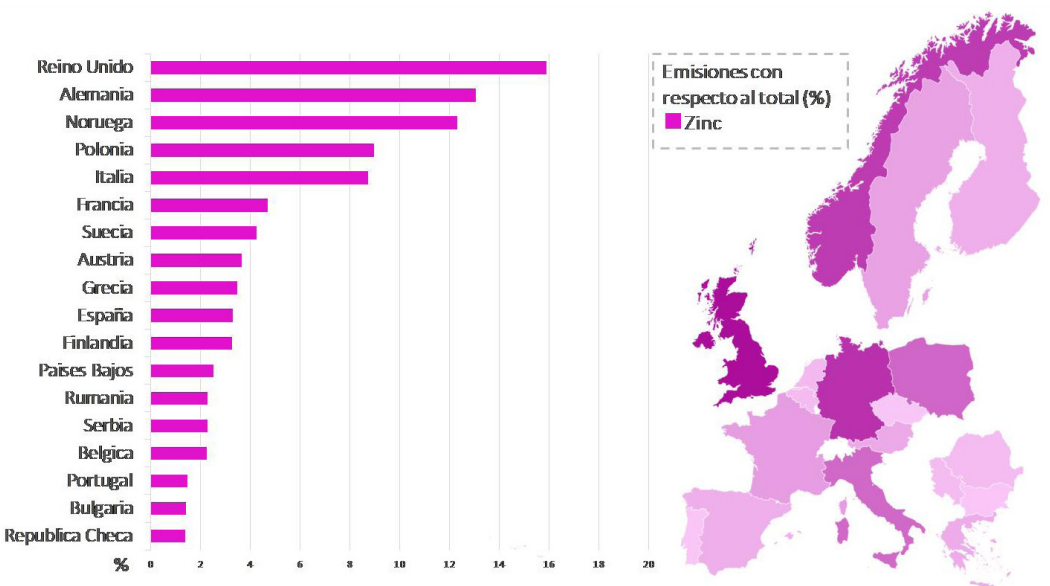

Figura 5. Emisiones de zinc. Contenido obtenido de E-PRTR. 

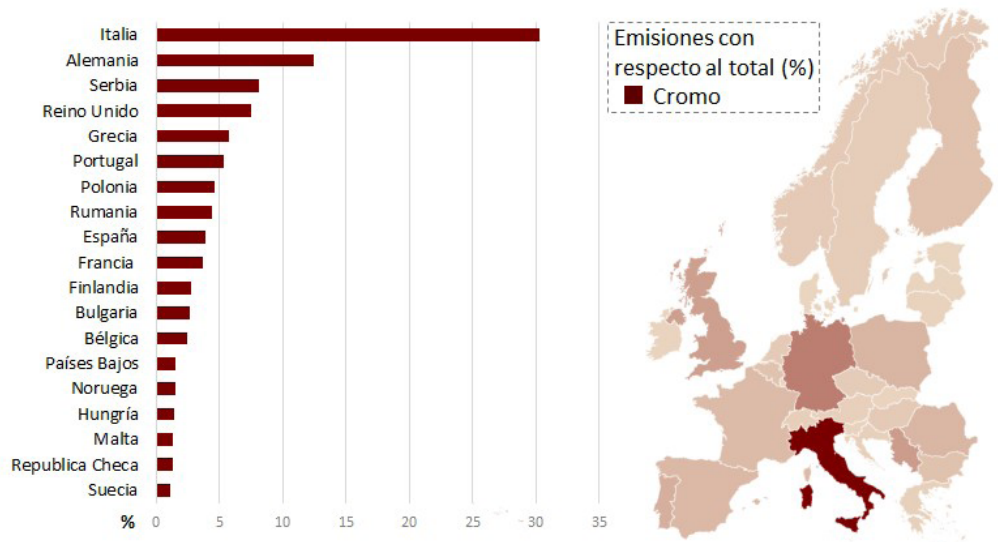

Figura 6. Emisiones de cromo. Contenido obtenido de E-PRTR.
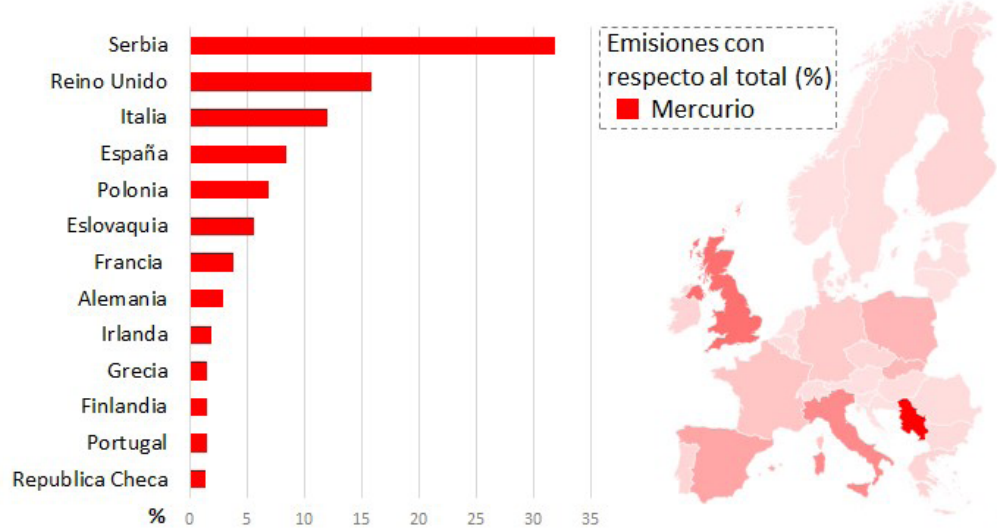

Figura 7. Emisiones de mercurio. Contenido obtenido de E-PRTR.

\subsection{Distribución espacial de las emisiones en España}

País Vasco, Cataluña, Comunidad Valenciana, Andalucía, Región de Murcia, Cantabria y Galicia son las regiones con mayor cantidad de puntos de emisión y municipios afectados por las emisiones de MP (Figura 8). La distribución espacial de puntos de emisión según el tipo de MP es desigual siendo el que mayor cantidad presenta el zinc, seguido del cromo, plomo, arsénico, cadmio y mercurio (Figura 9). País Vasco y Cataluña son las regiones con mayor cantidad de MP (Figuras 10 y 11) y, a su vez, son las regiones que albergan los municipios con niveles más altos de peligrosidad (Figura 9) seguidas de Andalucía, Castilla y León, Valencia, Aragón, Asturias, Islas Baleares, Murcia, Galicia y Cantabria. 


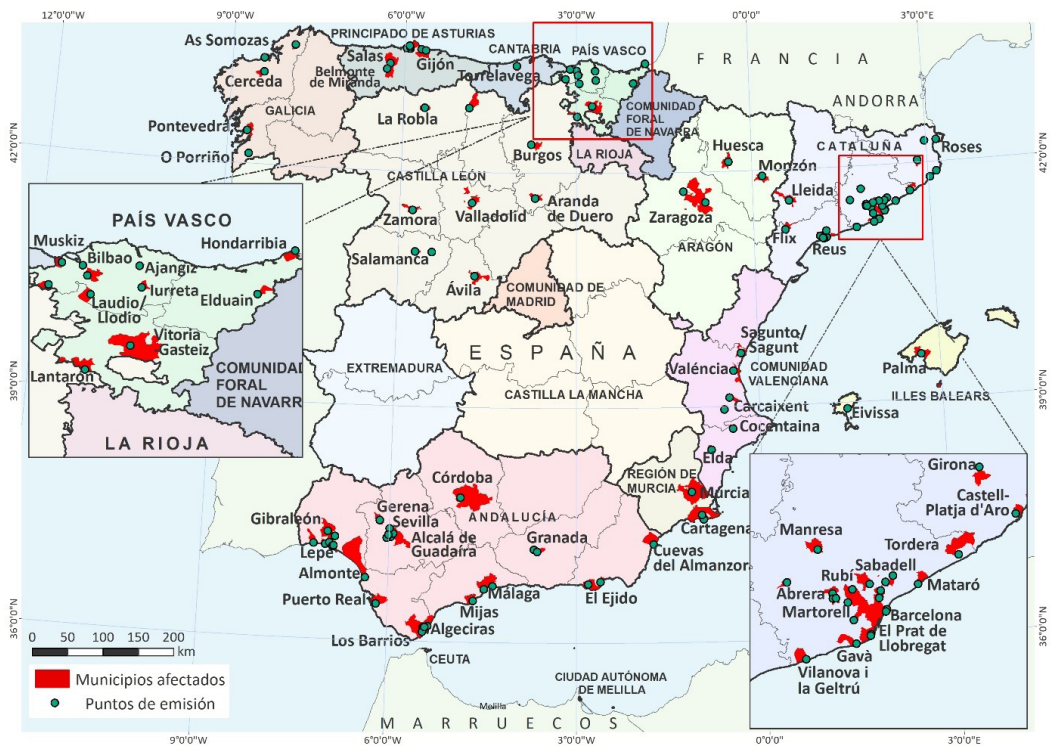

Figura 8. Puntos de emisión de MP y municipios afectados (2016).

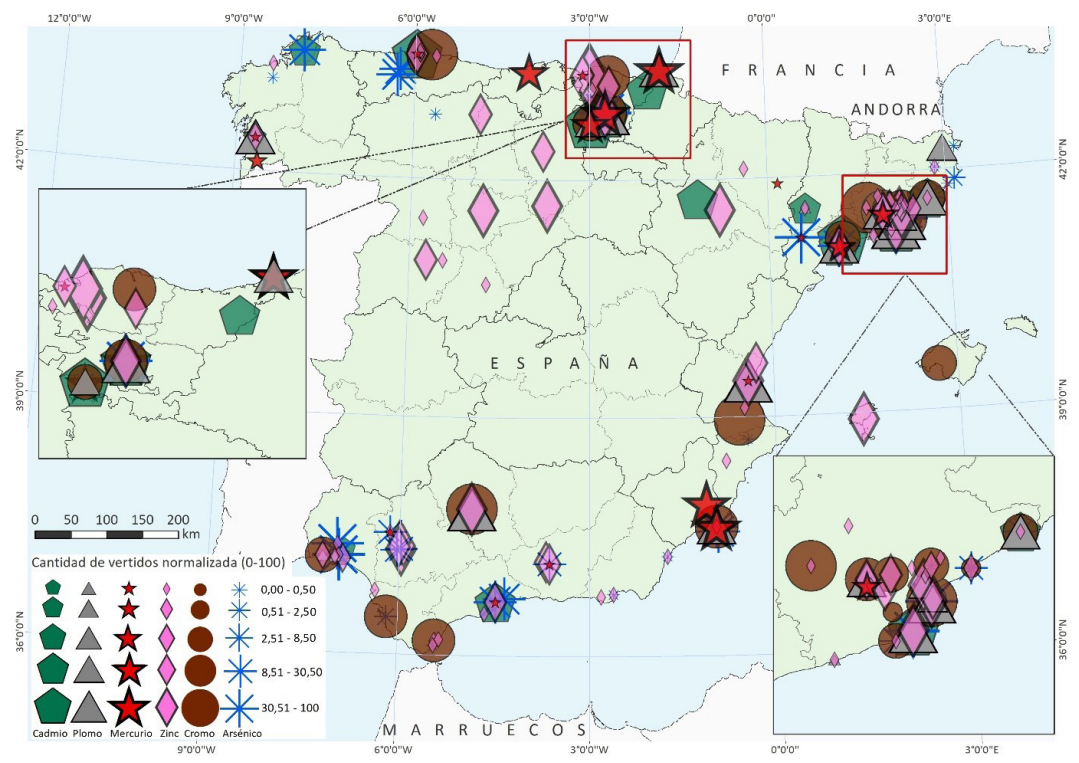

Figura 9. Distribución espacial de MP según el tipo de metal (2016). 


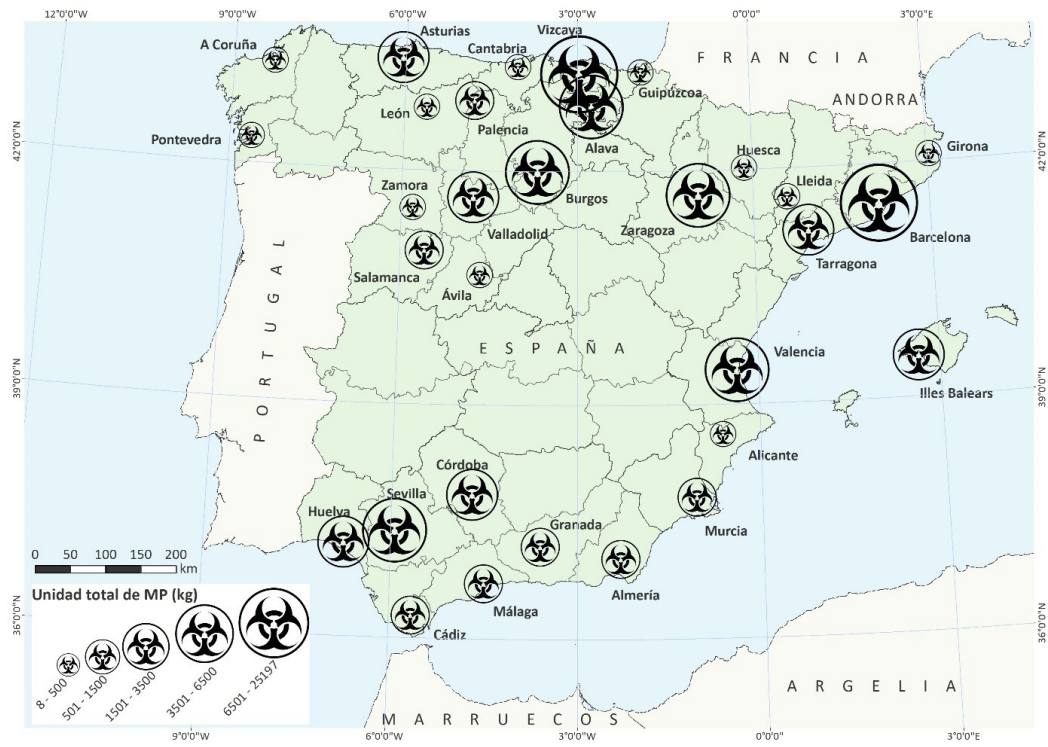

Figura 10. Emisión total de MP emitidos por provincias (2016).

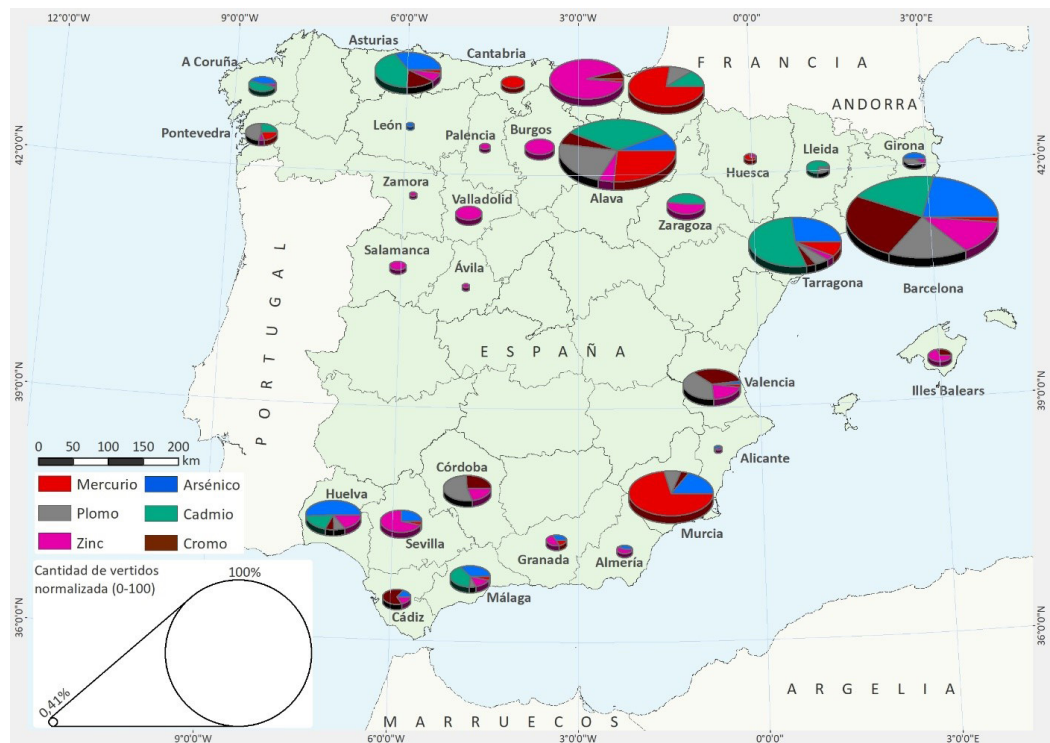

Figura 11. Emisión total de MP normalizada por provincias y tipo de metal (2016). 


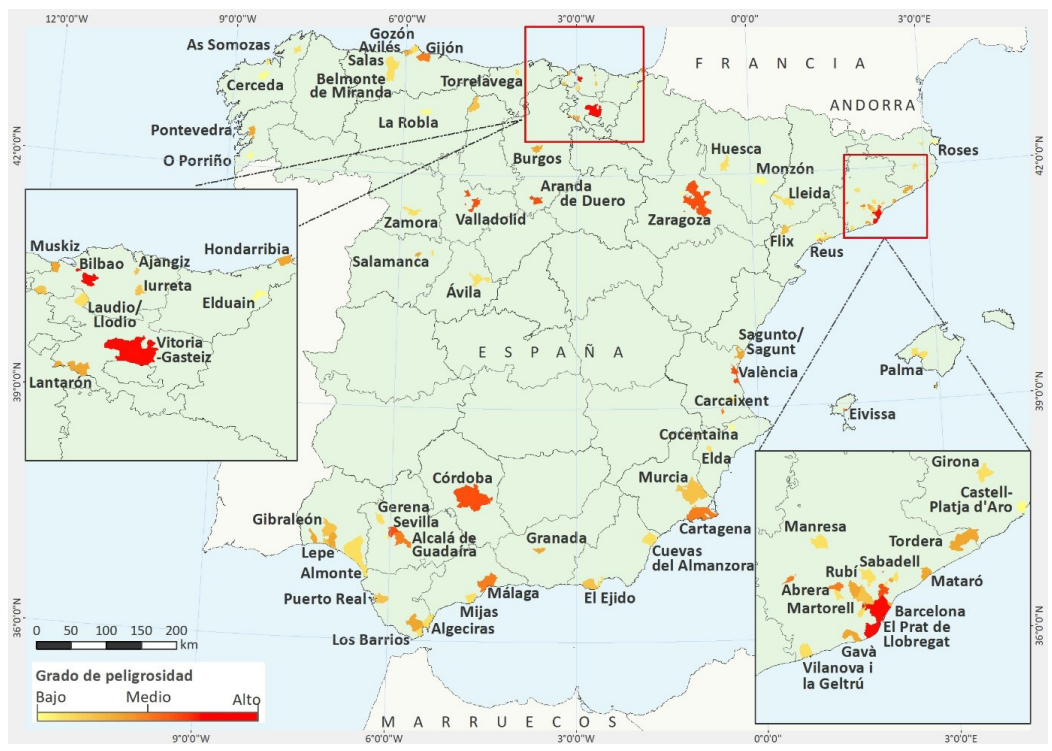

Figura 12. Grado de peligrosidad de los municipios expuestos a MT (2016).

\subsection{Evolución temporal de las emisiones de MP}

En la Figura 13 se puede apreciar, por un lado, el índice de producción industrial (IPI) de España entre 2007 y 2016 donde destaca el periodo de crisis económica que abarca de 2008 hasta 2012, y, por otro lado, la cantidad total de MP vertidos durante el mismo periodo. Analizando la tendencia del IPI y de las emisiones, se puede apreciar cómo la producción industrial y los vertidos de MP presentan un comportamiento similar con una tendencia paralela hasta el año 2013 momento en el que la producción industrial crece y las emisiones de MP se estabilizan. Este comportamiento hace que se planteemos si se están aplicando más medidas de control y como resultado las emisiones de MP disminuyan a la vez que la producción industrial aumenta lo cual interpretamos como un dato positivo.

En la Tabla 4 se muestra la cantidad anual y total acumulada de MP vertidos a lo largo de toda la serie analizada y en la Figura 11 se aprecia la variación por año. En verde se muestran los años donde los vertidos han descendido y en rojo los años en los que han aumentado. El MP que más se vierte a lo largo de toda la serie es el Zn, seguido de el $\mathrm{Cr}, \mathrm{Pb}, \mathrm{As}, \mathrm{Cd}$ y Hg. Como era de esperar, se observa que los MP considerados como menos peligrosos (zinc) registran mayor cantidad de vertidos mientras que los MP más peligrosos (mercurio) registran cantidades menores. 


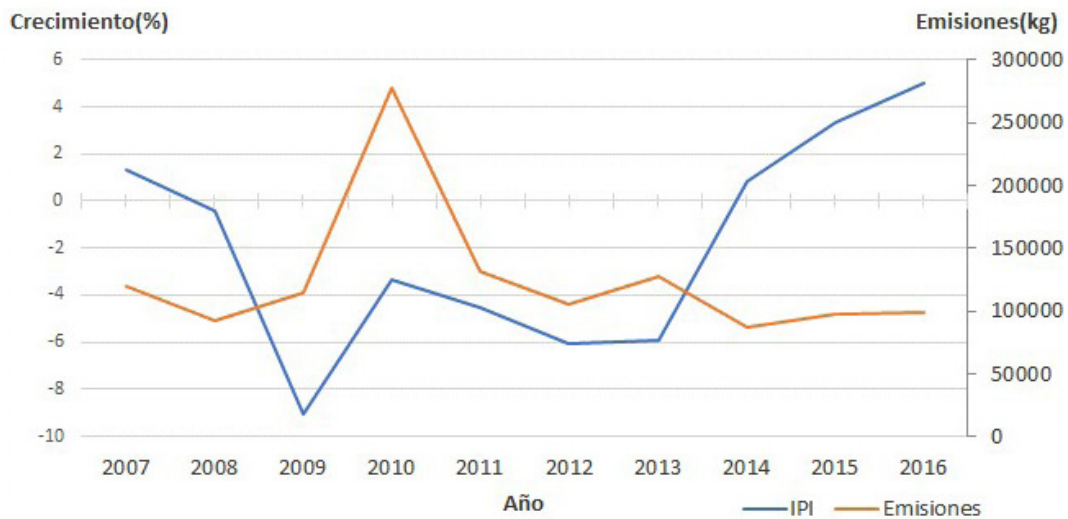

Figura 13. Índice de producción industrial de España.

Fuente: Banco Mundial de Datos.

Tabla 4. Cantidad y evolución de MP vertidos a lo largo de toda la serie analizada

\begin{tabular}{|c|c|c|c|c|c|c|c|c|c|c|c|c|c|c|c|c|c|c|}
\hline \multirow{3}{*}{ Año } & \multicolumn{18}{|c|}{ MP (kg) } \\
\hline & \multicolumn{3}{|c|}{ Ub (1) } & \multicolumn{3}{|c|}{$\mathrm{Ub}(20)$} & \multicolumn{3}{|c|}{ Ub (5) } & \multicolumn{3}{|c|}{ Ub (50) } & \multicolumn{3}{|c|}{ Ub (5) } & \multicolumn{3}{|c|}{ Ub (100) } \\
\hline & $\mathrm{Hg}$ & $\mathrm{Rg}$ & Ex & $P b$ & $\mathrm{Rg}$ & Ex & $C d$ & $\mathrm{Rg}$ & Ex & $\mathrm{Cr}$ & $\mathrm{Rg}$ & Ex & As & $\mathrm{Rg}$ & Ex & $Z n$ & $\mathrm{Rg}$ & Ex \\
\hline 2007 & 1019,5 & \begin{tabular}{|l|}
32 \\
\end{tabular} & 30,9 & 19266,5 & 24 & 39,1 & 4016,2 & 14 & 56,4 & 10164,9 & 13 & 14,6 & 4979,3 & 26 & 37,3 & 60592,0 & 53 & 10,4 \\
\hline 2008 & 999,6 & 33 & 29,3 & 2403,0 & 14 & 7,6 & 819,4 & 18 & 8,1 & 3757,0 & 25 & 2,0 & 3189,5 & 33 & 18,3 & 65591,0 & 80 & 7,2 \\
\hline 2009 & 828,1 & 26 & 30,9 & 4229,1 & 24 & 7,8 & 560,3 & 16 & 6,0 & 77540,0 & 23 & 66,4 & 2853,6 & 39 & 13,6 & 89240,0 & 85 & 9,5 \\
\hline 2010 & 432,5 & 23 & 17,8 & 7769,2 & 19 & 19,4 & 362,8 & 12 & 5,0 & 37410,7 & 24 & 30,2 & 2716,1 & 36 & 14,1 & 186106,0 & 95 & 18,6 \\
\hline 2011 & 130,5 & 24 & 4,4 & 4305,3 & 16 & 12,5 & 311,1 & 11 & 4,7 & 2490,6 & 14 & 2,6 & 1756,5 & 30 & 10,7 & 105345,0 & 91 . & 10,6 \\
\hline 2012 & 219,3 & 25 & 7,8 & 8823,3 & 19 & 22,2 & 2206,0 & 16 & 26,6 & 6564,1 & 20 & 5,6 & 2038,1 & 38 & 9,7 & 71218,0 & 87 & 7,2 \\
\hline 2013 & 319,7 & 26 & 11,3 & 10023,4 & 25 & 19,0 & 303,0 & 15 & 3,0 & 23469,3 & 36 & 12,0 & 1222,7 & 28 & 7,7 & 84117,0 & 92 & 8,1 \\
\hline 2014 & 259,3 & 18 & 13,4 & 3230,2 & 21 & 6,7 & 325,5 & 13 & 4,0 & 4112,8 & 20 & 3,1 & 1571,4 & 38 & 7,3 & 70534,0 & 95 & 6,4 \\
\hline 2015 & 266,0 & 20 & 12,3 & 2669,3 & 19 & 6,0 & 416,2 & 15 & 4,5 & 9496,4 & 22 & 7,6 & 2919,7 & 50 & 10,7 & 67511,0 & 94 & 6,2 \\
\hline 2016 & 334,9 & 19 & 16,6 & 2706,3 & 22 & 5,2 & 515,6 & 17 & 5,1 & 4161,5 & 26 & 2,2 & 2053,0 & 39 & 9,5 & 79390,0 & 80 & 8,9 \\
\hline Total & & 09,3 & & 6542 & 25,6 & & & 36,0 & & 1791 & 167,3 & & 252 & 299,8 & & 8796 & 44,0 & \\
\hline
\end{tabular}

La Figura 14 muestra la cantidad total normalizada de MP vertidos según el grado de peligrosidad. La evolución de vertidos experimenta cambios constantes a lo largo de toda la serie presentando transiciones bruscas en determinados años. La tendencia irregular (Figura 12) y poco clara a priori define una disminución de los vertidos. La tendencia de las emisiones presenta una evolución paralela a la evolución económica (Figura 10) habiendo una mayor cantidad de vertidos durante los años 2007, 2008 y 2009, seguido de un periodo donde las emisiones descienden coincidiendo con el periodo de crisis económica durante los años 2010, 2011 y 2012 para, finalmente, estabilizarse a partir del año 2013. 


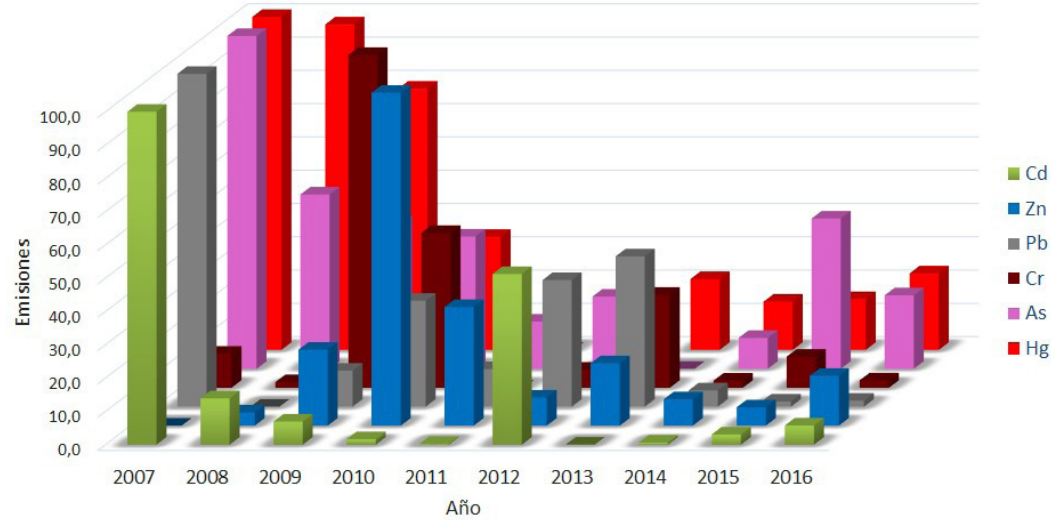

Figura 14. Evolución de las emisiones normalizadas de MP según el tipo de metal.

El análisis de la tendencia muestra unos resultados que difieren según la escala temporal del análisis. En un primer análisis, donde se analiza la totalidad de la serie (Figura 15) la tendencia muestra una línea descendente cuyo comportamiento podría determinar que existe una situación de mejora continuada con el paso de los años.

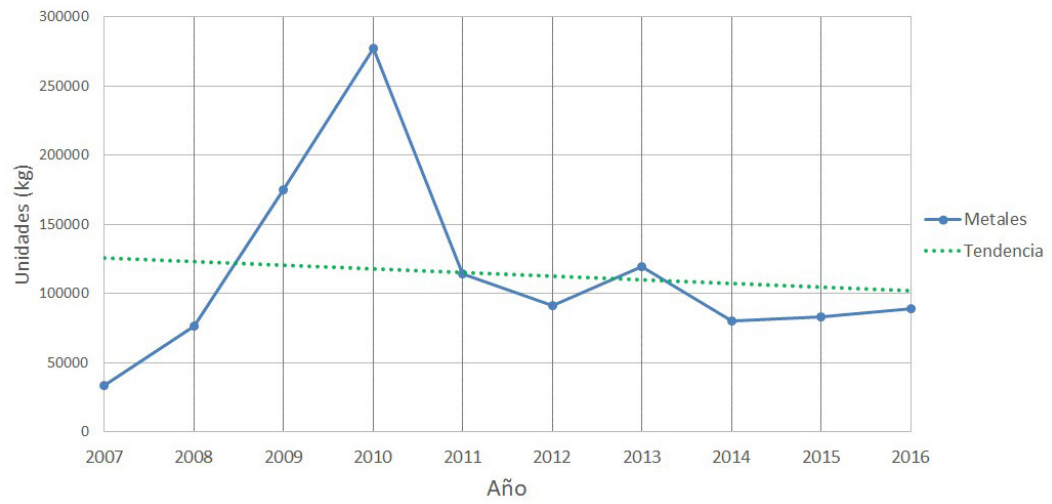

Figura 15. Tendencia de las emisiones de MP.

Sin embargo, analizando la tendencia anualmente (Tabla 5), se observa que en determinados años las emisiones aumentan o se reducen considerablemente con respecto al año anterior denotando una tendencia no lineal e irregular 
cuyo comportamiento refleja una situación inestable con periodos de mejoría y empeoramiento de forma intermitente. Esto puede deberse a varios factores cómo la posibilidad de que en determinados años el número de registros totales de empresas que hayan realizado la declaración de emisiones sea mayor o menor dado la voluntariedad del registro dependiendo de si han superado el umbral de emisiones o no.

Tabla 5 . Evolución anual del total de MP vertidos

\begin{tabular}{|c|c|c|c|c|c|c|c|c|c|c|}
\hline Año & 2007 & 2008 & 2009 & 2010 & 2011 & 2012 & 2013 & 2014 & 2015 & 2016 \\
\hline Total & 33447,6 & 76759,5 & 175251 & 277490 & 114339 & 91068,8 & 119455 & 80033,16 & 83278,6 & 89161,2 \\
\hline Evolución & & 1. 56,4 & I. 56,2 & 1) 36,8 & I $-142,7$ & I $-25,6$ & 1) 23,8 & $-49,3$ & 3,9 & 1 \\
\hline
\end{tabular}

\section{Discusión}

El número total de empresas que registran emisiones de MP varía cada año habiendo en España únicamente cuatro que presentan registros en toda la serie analizada. Dado que los registros son obligatorios solo si se superan los umbrales de emisión, el hecho de que en unos años se reduzca el número total de empresas declarantes puede ser un aspecto positivo puesto que podría suponer se están reduciendo los vertidos de MP o, por otro lado, como un aspecto negativo, que las empresas no cumplen continuadamente con los registros anuales de emisiones incumpliendo con la normativa.

Los municipios con mayor grado de exposición a los MP se encuentran lógicamente en las regiones de mayor desarrollo industrial y que concentran la mayoría de las empresas que emiten MP. La mayoría de ellos se localizan en las provincias de País Vasco y Cataluña, seguido de las de la Comunidad Valencia, Andalucía, Cantabria y Galicia.

\subsection{Limitaciones}

Los datos de emisiones no presentan series suficientemente largas como para determinar si la tendencia es de signo positivo o negativo. Además, recordemos, los registros no cubren la serie completa más que en algún caso muy concreto. A pesar de las citadas limitaciones, consideramos que las aportaciones del estudio son relevantes y sientan las bases de futuros análisis sobre una problemática insuficientemente abordada en la literatura científica.

\section{Conclusión}

Las limitaciones señaladas no han impedido alcanzar los objetivos planteados. A partir de la información recogida en la revisión bibliográfica se ha conseguido cuantificar la peligrosidad la toxicidad de los vertidos de MP y a partir de la integración de la información en un SIG, se ha producido una cartografía de concentraciones de MP. 
A partir de la cartografía producida, tanto a nivel nacional como europeo, se han podido comparar las emisiones de MP de España con las de los países de su entorno y profundizar en el conocimiento sobre la distribución espacial de los puntos de emisión y cantidades vertidas de MP.

Por último, con severas limitaciones debido a la inconsistencia de la serie de datos, se ha estudiado la evolución de los vertidos de MP.

Como se ha señalado en la introducción, el grado y tiempo de exposición, así como el tipo de químico, son los factores clave para caracterizar y determinar la peligrosidad de los MP. Podemos afirmar que el mercurio $(\mathrm{Hg})$ es el metal más tóxico y nocivo, seguido del plomo (PB), cadmio (Cd), cromo (Cr), arsénico (As) y zinc (Zn). Este aspecto es crucial a la hora de determinar qué áreas presentan una mayor peligrosidad según el tipo y cantidad de metal al que se exponen. A su vez, se considera que existe una correlación entre la toxicidad y los umbrales de emisión sobrepasados y, por tanto, la cantidad de MP vertidos por año. Por lo general cuanto menos letal es un MP, su umbral de emisiones es menos restrictivo y mayor es la cantidad que se vierte, y cuanto más letal es un MP, a la inversa.

El análisis espacial muestra a priori que, a nivel nacional, España es uno de los países de Europa (miembros de la E-PRTR) cuyo nivel de emisiones de MP es medio-alto. A nivel local, existe un obvio paralelismo entre la distribución espacial de los MP y las áreas de mayor concentración industrial localizadas principalmente en el País Vasco, Cataluña, Valencia y Andalucía.

El análisis temporal determina que existe una tendencia no lineal donde la situación tiende a evolucionar de manera irregular y sin que exista una mejora aparente del fenómeno. A su vez, se aprecia una relación entre la cantidad de emisiones y la situación socioeconómica coincidiendo los periodos con menos emisiones con el inicio y primeros años de la crisis económica (2008) y los periodos con más emisiones con los años de recuperación económica.

Estos resultados constituyen una primera aproximación al análisis cartográfico de los vertidos de MP en España y sientan las bases para futuros desarrollos.

\section{Bibliografía}

Achparaki, M.; Thessalonikeos, E.; Tsoukali, H.; Mastrogianni, O.; Zaggelidou, E.; Chatzinikolaou, F.; Vasilliades, N. \& Raikos, N. (2012). Heavy metals toxicity. Aristotle University Medical Journal, 39(1), 29-34.

Atta, E. R.; Zakaria, K. M. \& Ibrahim, M. S. (2016). Assessment of the Heavy Metals and Natural Radioactivity in Phosphate Mines and Occupational Health Effects at Some Egyptian Regions. Journal of Environmental Protection, 07(11), 1657-1669. https://doi.org/10.4236/jep.2016.711135.

Comisión Europea (2006). Guía para la implantación del E-PRTR. European Evironmental Agency. Recuperado de https://prtr.eea.europa.eu/\#/downloadguidance.

Cuihua Chen; Shijun Ni; Chengjiang Zhang \& Binbin He (2007). Assessing spatialtemporal variation of heavy metals contamination of sediments using GIS 3D spatial analysis methods in Dexing mines, Jiangxi province, China. 
IEEE International Geoscience and Remote Sensing Symposium, 1650-1653. https://doi.org/10.1109/IGARSS.2007.4423132.

European Parlianment y The Council of the European Union (2006). Regulation (EC) No $166 / 2006$ of the European Parliament and of the Council. Official Journal of the European Union: L. 33/1. Recuperado de https://www.eea.europa.eu/policydocuments/regulation-ec-no-166-2006.

European Union (2010). EU Directive 2010/75/EY.

European Commission (2006). The European Pollutant Release and Transfer Register (E-PRTR) Regulation (166/2006/EC).

Ismail, T. N. H. T.; Adon, R.; Diman, S. F. \& Wijeyesekera, D. C. (2013). Innovative green technology and products meeting geo-environmental challenges. Procedia Engineering, 53, 104-115. https://doi.org/10.1016/j.proeng.2013.02.016.

Jaishankar, M.; Tseten, T.; Anbalagan, N.; Mathew, B. B. \& Beeregowda, K. N. (2014). Toxicity, mechanism and health effects of some heavy metals. Interdisciplinary Toxicology, 7(2), 60-72. https://doi.org/10.2478/intox-2014-0009.

Londoño-Franco, L. F.; Londoño-Muñoz, P. T. \& Muñoz-Garcia, F. G. (2016). Los riesgos de los metales pesados en la salud humana y animal. Biotecnoloía en el Sector Agropecuario y Agroindustrial, 14(2), 145-153. https://doi.org/10.18684/BSAA(14)145-153.

Moslen, M. \& Miebaka, C.A. (2018). Consumption of Shrimps (Penaeus sp) and Health Risk Evaluation due to Heavy metal contamination in the upper reaches of the Bonny Estuary, Nigeria. Perspectives in Ecotoxicology, editado por Lenin Media Private Limited, Delhi, India Ltd., 104-113.

Morais, S.; García e Costa, F. \& de Lourdes P. M. (2012). Heavy Metals and Human Health. Environmental Health - Emerging Issues and Practice, Prof. Jacques Oosthuizen (ed.), InTech. Recuperado de http://www.intechopen.com/books/ environmental-health-emerging-issuesand-practice/heavy-metals-andhuman-health

Peng, M.; Li, R.; Cui, X.; Ding, W. \& Zhao, X. (2011). The preliminary application of WebGIS in assessing environmental impact on human health. 19th Interna-tional Conference on Geoinformatics, 1-4. https://doi.org/10.1109/Geolnformatics.2011.5980742.

Sánchez-Díaz, G.; Escobar, F.; Badland, H.; Arias-Merino, G.; de la Paz, M. P. \& AlonsoFerreira, V. (2018). Geographic Analysis of Motor Neuron Disease Mortality and Heavy Metals Released to Rivers in Spain. International Journal of Environmental Research and Public Health, 15(11). https://doi.org/10.3390/ijerph15112522.

Tchounwou, P. B.; Yedjou, C. G.; Patlolla, A. K. \& Sutton, D. J. (2012). Heavy Metal Toxicity and the Environment. Experientia Supplementum, 101, 133-164.

Yu, M.-H. (2005). Biological and health effects of pollutants. Environmental toxicology. Recuperado de https://search.proquest.com/docview/231475732/fulltext/ 754196BA34884754PQ/1?accountid=14475. 